\title{
Lipid starvation and hypoxia synergistically activate ICAM1 and multiple genes in an Sp1-dependent manner to promote the growth of ovarian cancer
}

Shiro Koizume ${ }^{1 *}$, Shin Ito ${ }^{1}$, Yoshiyasu Nakamura' ${ }^{1}$ Mitsuyo Yoshihara' ${ }^{1}$ Mitsuko Furuya ${ }^{2}$, Roppei Yamada', Etsuko Miyagi ${ }^{3}$, Fumiki Hirahara ${ }^{3}$, Yasuo Takano ${ }^{1}$ and Yohei Miyagi ${ }^{* *}$

\begin{abstract}
Background: Elucidation of the molecular mechanisms by which cancer cells overcome hypoxia is potentially important for targeted therapy. Complexation of hypoxia-inducible factors (HIFs) with aryl hydrocarbon receptor nuclear translocators can enhance gene expression and initiate cellular responses to hypoxia. However, multiple molecular mechanisms may be required for cancer cells to adapt to diverse microenvironments. We previously demonstrated that a physical interaction between the ubiquitously expressed transcription factor Sp1 and HIF2 is a major cause of FVII gene activation in poor prognostic ovarian clear cell carcinoma (CCC) cells under hypoxia. Furthermore, it was found that FVII activation is synergistically enhanced when serum-starved cells are cultured under hypoxic conditions. In this study, we investigated whether HIFs and transcription factor Sp1 cooperate to activate multiple genes in CCC cells under conditions of serum starvation and hypoxia (SSH) and then contribute to malignant phenotypes.
\end{abstract}

Methods: To identify genes activated under hypoxic conditions in an Sp1-dependent manner, we first performed cDNA microarray analyses. We further investigated the molecular mechanisms of synergistic gene activations including the associated serum factors by various experiments such as real-time RT-PCR, western blotting and chromatin immunoprecipitation. The study was further extended to animal experiments to investigate how it contributes to CCC progression in vivo.

Results: ICAM1 is one such gene dramatically induced by SSH and is highly induced by SSH and its synergistic activation involves both the mTOR and autonomously activated TNFa-NFKB axes. We identified long chain fatty acids (LCFA) as a major class of lipids that is associated with albumin, a serum factor responsible for synergistic gene activation under SSH. Furthermore, we found that ICAM1 can be induced in vivo to promote tumor growth.

Conclusion: Sp1 and HIFs collaborate to activate genes required for the adaptation of CCC cells to severe microenvironments, such as LCFA starvation and hypoxia. This study highlights the importance of transcriptional regulation under lipid starvation and hypoxia in the promotion of CCC tumor growth.

Keywords: Hypoxia, Sp1, Lipid starvation, ICAM1, Ovarian cancer

\footnotetext{
* Correspondence: skoizume@gancen.asahi.yokohama.jp;

miyagi@gancen.asahi.yokohama.jp

${ }^{1}$ Molecular Pathology and Genetics Division, Kanagawa Cancer Center Research Institute, 2-3-2 Nakao, Asahi-ku, Yokohama 241-8515, Japan Full list of author information is available at the end of the article
} 


\section{Background}

Cancer cells adapt to hypoxic microenvironments using aberrant vasculature to enable tumor growth. Hypoxiainducible factors (HIFs) $1 \alpha$ and $2 \alpha$ are major transcription factors required for adaptive responses to hypoxia. HIFs in complex with the aryl hydrocarbon receptor nuclear translocator (ARNT) can bind hypoxia response elements (HREs) of target genes, where they act to enhance transcription [1]. This conventional mechanism regulates genes such as VEGF and $H O$ to improve relatively mild hypoxic conditions [2,3]. However, many tumor types, especially those of pancreatic and cervical origin, are known to experience more severe hypoxic stresses [4].

To respond to dynamic changes in hypoxic conditions combined with variable nutrient, growth factor, and hormone environments, tumor cells employ multiple stress response mechanisms. In addition to HIF signaling, endoplasmic reticulum (ER) stress followed by the unfolded protein response (UPR) is another cellular response involved in adaptation to severe hypoxia [3]. The UPR can stimulate the transcription of additional genes required for tumor survival and growth.

Mammalian target of rapamycin (mTOR) signaling is also important for adaptation of cancer cells to hypoxia. mTOR is a serine/threonine kinase and a regulator of protein expression at the translational level [5]. mTOR is activated via phosphorylation of its serine residues, and its activation regulates levels of cellular nutrients, energy availability, and growth factors [6]. In general, mTOR becomes inactivated in hypoxic tumor tissues. However, it is known that mTOR dysregulation promotes the translation of HIF1 [5] and cooperates in responses to hypoxic conditions, suggesting that the interaction between the HIF- and mTOR-signaling pathways is involved in adaptation to hypoxia.

We previously demonstrated that the FVII gene, which encodes blood coagulation factor VII (fVII) [7], can be induced in ovarian clear cell carcinoma (CCC) cells in response to hypoxia $[8,9]$. Ectopic expression of this procoagulant promotes phenotypic changes in cancer cells $[8,10]$. We also demonstrated that a physical interaction between the ubiquitously expressed transcription factor Sp1 [11] and HIF2 is a major cause of FVII activation in CCC cells under hypoxic conditions [12]. Furthermore, FVII activation is synergistically enhanced via a UPRindependent pathway when serum-starved cells are cultured under hypoxic conditions, explaining why thrombosis frequently occurs in CCC patients [12]. However, little is known of the mechanisms underlying this synergism.

Ovarian cancer is most the malignant of the gynecological neoplasms $[13,14]$. CCC in particular is highly resistant to chemotherapy and has a poor prognosis
[14]. We hypothesized that genes regulated by the above-described Sp1-dependent mechanism are responsible for adaptive responses to hypoxia with a severity and/or duration that is characteristic of CCC tissues. The aim of this study was to test this hypothesis. We first tested whether multiple genes are activated under hypoxia in an Sp1-dependent manner in CCC cells and whether such transcriptional activations occur synergistically during serum starvation. We further investigated the molecular mechanisms of synergistic gene activation, including regulation by associated serum factors. The study was further extended to investigate ICAM1 gene expression in vivo and its contribution to CCC progression.

\section{Results}

Sp1 and HIFs cooperate to activate multiple genes under hypoxia

To identify genes activated under hypoxic conditions in an Sp1-dependent manner, we first performed cDNA microarray analyses. We used $\mathrm{CoCl}_{2}$ treatment under normoxia, as we found previously that FVII induction with this hypoxia-mimicking agent is higher than induction observed by maintaining cells in $1 \% \mathrm{O}_{2}$ [12]. A CCC cell line (OVSAYO) was first transfected with Sp1-siRNA (Sp1-si) or negative control siRNA (NS-si) and then cultured with $\left(\rightarrow \mathrm{CoCl}_{2}\right)$ or without $\mathrm{CoCl}_{2}$. Western blotting showed that Sp1 knockdown did not affect HIF induction (Figure 1a). Basal promoter activity of VEGF [15] and HO [16] genes can be regulated by $\mathrm{Sp} 1$. However, the expression ratios of these $\mathrm{HRE}$-dependent genes in $\mathrm{CoCl}_{2}$ treated cells vs. non-treated cells were largely unchanged between control cells (NS-si $\rightarrow \mathrm{CoCl}_{2}$ treatment/NS-si) and $\mathrm{Sp} 1$-silenced cells $\left(\mathrm{Sp} 1-\mathrm{si} \rightarrow \mathrm{CoCl}_{2}\right.$ treatment/NS-si and $\mathrm{Sp} 1-\mathrm{si} \rightarrow \mathrm{CoCl}_{2}$ treatment/Sp1-si) (Figure 1b). In contrast, expression of multiple genes in the presence of $\mathrm{CoCl}_{2}$ was decreased by $\mathrm{Sp} 1$ silencing (Figure $1 \mathrm{~b}$ and Additional file 1: Table S1). Among them, the GAPDH and TGFB genes have also been reported to be HREdependent $[17,18]$.

We tested whether genes activated in an Sp1-dependent manner were also inducible under real hypoxia $\left(1 \% \mathrm{O}_{2}\right)$ by focusing on 4 genes (ICAM1 [19], KLF6 [20], JUN [21], and $A R$ [22]; Figure $1 \mathrm{~b})$, as their upregulation by direct interactions with HIFs has not been reported and their basal promoter activities are regulated by Sp1. Real-time reverse-transcription polymerase chain reaction (RT-PCR) analysis revealed that ICAM1, KLF6, and JUN genes were weakly upregulated under hypoxia and in the presence of fetal calf serum (FCS) (Figure 2a, FCS + H) compared with normoxic conditions (Figure 2a, FCS $+\mathrm{N}$ ). RT-PCR results also showed that siRNA-mediated knockdown of HIFs, particularly HIF1, impaired hypoxic induction of these genes (Figure 2b). We confirmed by chromatin immunoprecipitation (ChIP) that Sp1 and HIFs bind authentic 

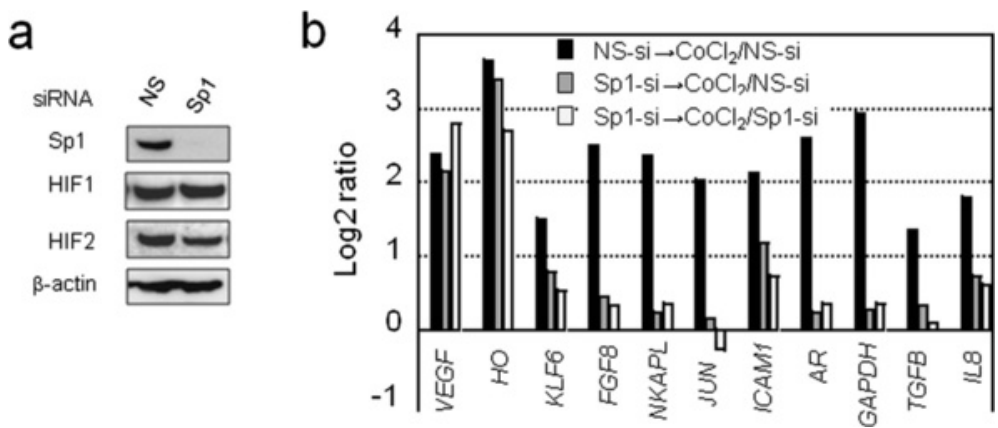

Figure 1 cDNA microarray analysis of genes activated by $\mathrm{CoCl}_{2}$ stimulation in an Sp1-dependent manner. a) western blot analysis of lysates from cells transfected with non-specific (NS) or Sp1 siRNA. b) log2 ratio of mRNA levels transfected with NS- or Sp1-siRNAs and then cultured for $24 \mathrm{~h}$. Cells were further cultured for an additional $4 \mathrm{~h}$ under stimulation with $\mathrm{CoCl}_{2}(500 \mu \mathrm{M})$ or treatment with a vehicle control.

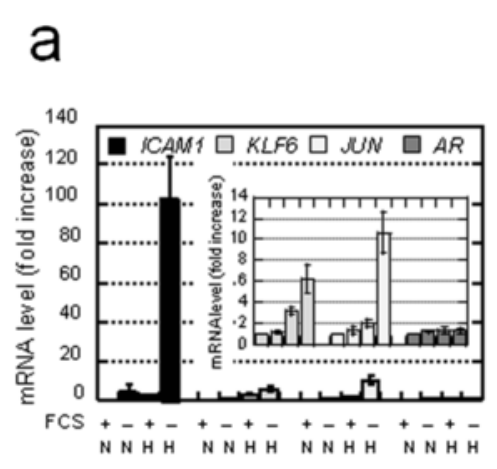

C

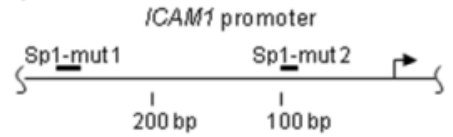

Sp1-mut 1 5'-GGGAGGCGTGGAGGCCGGGAGCAG-3'

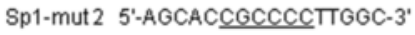

e

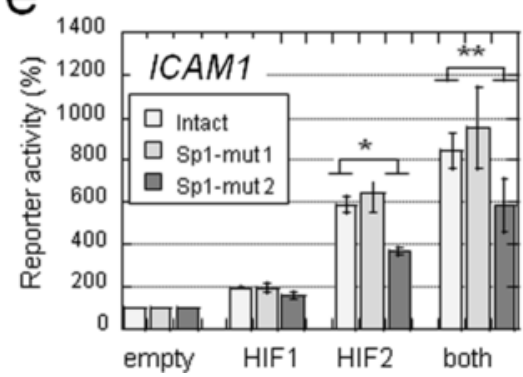

b
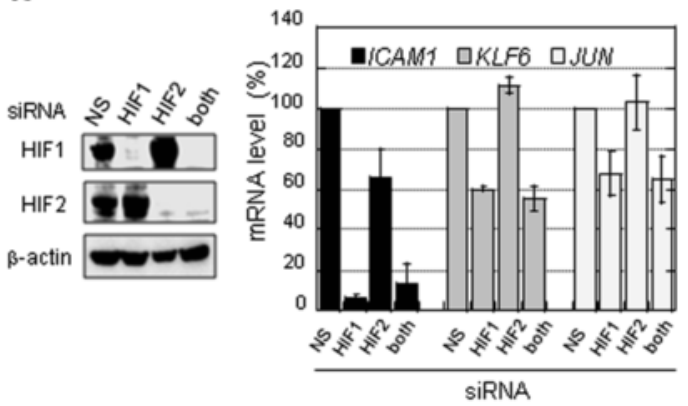

d

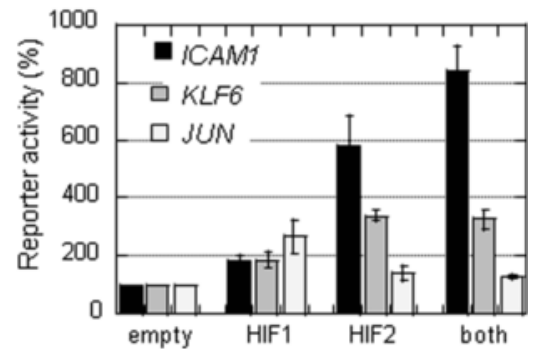

Figure $2 \mathrm{Sp} 1$ and HIFs contribute to the synergistic activation of multiple genes in OVSAYO cells under SSH. a) Synergistic activation of genes under SSH. N and $\mathrm{H}$ indicate normoxia $\left(20 \% \mathrm{O}_{2}\right.$ for $\left.16 \mathrm{~h}\right)$ and hypoxia $\left(1 \% \mathrm{O}_{2}\right.$ for $\left.16 \mathrm{~h}\right)$, respectively. Data shown represent the mean $(n=3) \pm S D$. b) Activation of multiple genes under hypoxia is dependent on HIFs. Western blotting is also shown for HIFs. Data shown are the mean $(n=3) \pm S D$. c) The ICAM1 promoter region and predicted Sp1 binding sites (ref. 19). PCR-amplicons generated in the reporter gene assay are shown in Additional file 2: Figure S1a. Bold bars: mutated sites (Sp1-mut 1 and 2); underlined nucleotide sequences: regions deleted by mutagenesis. $\mathbf{d}$, e) Luciferase reporter gene assay. Plasmids were co-transfected with the indicated vectors, and luciferase activities were measured at $24 \mathrm{~h}$ post-transfection. Luciferase activities measured in cells transfected with the empty vector were defined as $100 \%$. Data shown are the mean $(n=3) \pm S D .{ }^{*} P=0.0018 ;{ }^{* *} P=0.075$. 
promoter regions of these 3 genes in OVSAYO cells (Additional file 2: Figure S1a). Furthermore, luciferase reporter gene analysis revealed that the expression of HIFs can activate the ICAM1 promoter more efficiently than the KLF6 and JUN promoters (Figure 2c and d). Unlike knockdown experiments targeting endogenous genes, the effect on ICAM1 promoter activation was predominant for HIF2. Further experiments demonstrated that activation of the ICAM1 promoter by HIF2 is impaired when a known Sp1 binding site is mutated (Figure 2c and e). ChIP assay results showed that ARNT weakly bound to the ICAM1 promoter region, but this binding was not enhanced in response to $\mathrm{CoCl}_{2}$ treatment (Figure $3 \mathrm{a}$ and $\mathrm{b}$ ). However, HIF2 became bind the promoter region by $\mathrm{CoCl}_{2}$ exposure (Figure 3c). As expected, ARNT was found to bind the VEGF-HRE region in response to $\mathrm{CoCl}_{2}$ treatment (Figure 3a).

Previously, we demonstrated that the PAS domains of HIF2 are required for its association with Sp1 [12]. Thus, here we tested whether the PAS domains are important for activation of the ICAM1 promoter by HIF2. Luciferase reporter gene analysis revealed that HIF2-driven activation of the ICAM1 promoter was largely impaired when the domain-deleted HIF2 mutant (HIF2 $\triangle \mathrm{PAS}$; 351-870 construct; ref. 12) was expressed instead of the full-length HIF2 (Figure 3d and e). Collectively, these results demonstrate that $\mathrm{Sp} 1$ and HIF2 can cooperate to mediate ICAM1 induction in a HRE-independent manner.

\section{Sp1-dependent gene activation under hypoxia is synergistically enhanced by serum deprivation}

Serum starvation is a crucial component of ischemia [23]. Hence, we next tested whether Sp1-dependent activation of the selected genes is enhanced under serum starvation and hypoxia (SSH), as in the case of FVII [12]. We found that ICAM1, KLF6, and $J U N$, but not $A R$, are synergistically activated under SSH. Notably, ICAM1 expression was dramatically induced (Figure 2a, FCS- H). This robust expression under $\mathrm{SSH}$ may be characteristic of CCC cells as this was also true in an additional CCC cell line, OVISE (Additional file 2: Figure S1b). Other ovarian cancer cells of different histological origin did not exhibit such highly synergistic activation of genes (data not shown). We found that the synergistic ICAM1 gene induction was decreased by $\mathrm{Sp} 1$ knockdown at mRNA (Additional file 2: Figure S2a) and protein (Additional file 2: Figure S2b) levels. This Sp1 dependence was also confirmed for KLF6 and JUN induction, although such SSH-specific effects were not observed for $V E G F$ expression (Additional file 2: Figure S2a).

The synergism involved in ICAM1 induction was evident. It has been reported that overexpression of ICAM1 protein correlates with cancer prognosis [24]. Thus, we
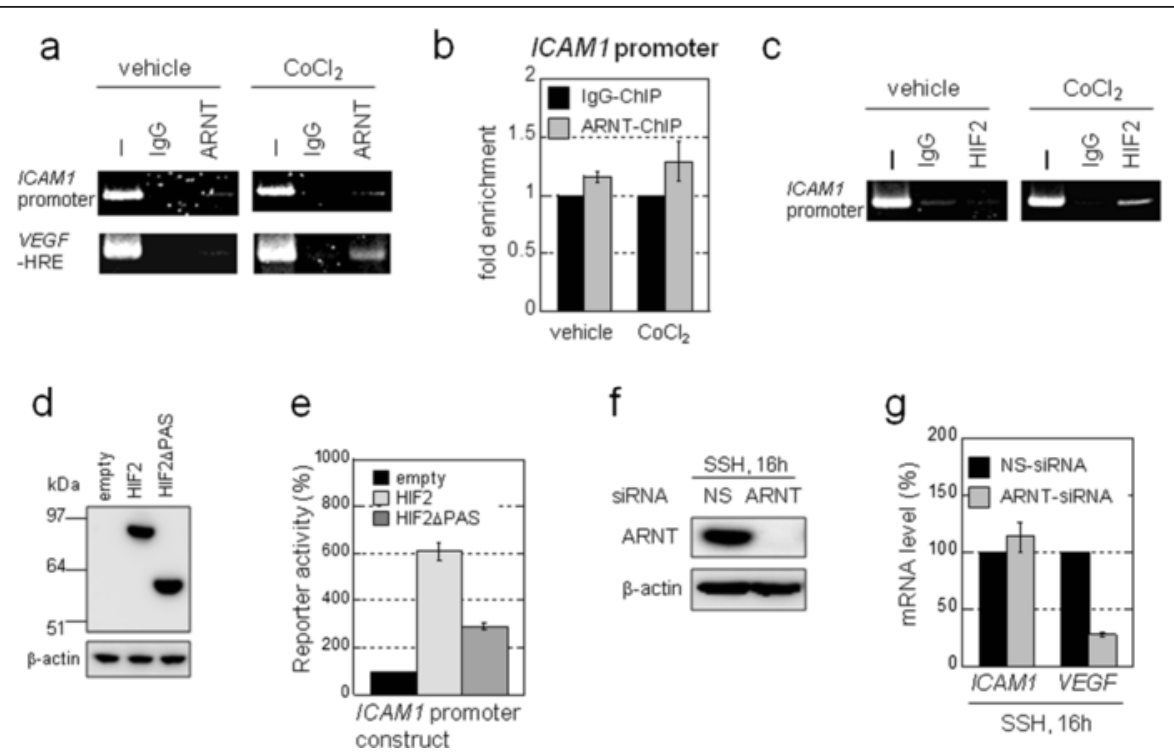

Figure 3 Synergistic activation of the ICAM1 gene is independent of ARNT. a) ChIP analysis of ARNT binding to gene regulatory regions of the indicated genes. Cells were cultured for $4 \mathrm{~h}$ under stimulation with $\mathrm{CoCl}_{2}(500 \mu \mathrm{M})$ or treatment with a vehicle control. I: Input control lysate without immunoprecipitation. IgG: negative control using lgG. b) Results in a) were quantitatively estimated by real-time PCR analysis. c) ChIP analysis of HIF2 binding to the ICAM1 promoter region. Cells were treated as in a). d) The ICAM1 promoter construct (Figure 2e) was co-transfected with the indicated expression vectors. After $24 \mathrm{~h}$, expression of indicated proteins was confirmed by western blotting. e) Luciferase activities were measured at $24 \mathrm{~h}$ post-transfection. Luciferase activities measured in cells transfected with the empty vector were defined as 100\%. Data shown are the mean $(n=3) \pm S D$. f) Western blot analysis of lysates from OVSAYO cells transfected with non-specific (NS) or ARNT siRNA and then cultured under SSH for an additional 16 h. g) Real-time RT-PCR analysis of ICAMI and VEGF gene expressions in OVSAYO cells transfected with NS or ARNT siRNA and then cultured under SSH conditions for an additional $16 \mathrm{~h}$. Data shown are the mean $(n=3) \pm$ SD. 
focused our study primarily on this gene. We found that synergistic ICAM1 expression was decreased by silencing HIFs (Additional file 2: Figure S2c). However, unlike in hypoxic conditions with serum stimulation, both HIF1 and HIF2 contributed equally to synergistic ICAM1 induction. Moreover, real-time RT-PCR analysis revealed that synergistic activation of the ICAM1 gene under SSH conditions is not affected by ARNT knockdown, while $V E G F$ expression was largely diminished (Figure $3 \mathrm{f}$ and $\mathrm{g}$ ). These results demonstrate that ICAM1 gene induction under SSH can be mediated via ARNT-independent interactions between Sp1 and HIFs within the gene promoter region.

\section{UPR does not contribute to synergistic ICAM1 activation} A UPR marker CHOP is induced in OVSAYO cells cultured under SSH (Additional file 2: Figure S3a). Thus, we tested whether expression of the ICAM1 gene under hypoxia with or without FCS treatment was enhanced by simultaneous treatment with the UPR-inducing agent, tunicamycin (Tun). Tun induced CHOP under hypoxia with or without FCS (Additional file 2: Figure S3b and c) and suppressed ICAM1 expression (Additional file 2: Figure S3d and e). The expression levels of Sp1 and HIFs were not significantly altered (Additional file 2: Figure S3b), suggesting that the synergistic activation is not mediated by the UPR, as is the case with FVII [12].

\section{mTOR is involved in synergistic gene activation under SSH}

Western blotting showed that mTOR activation was suppressed in OVSAYO cells cultured under SSH, as phosphorylation at Ser2448 was decreased compared that observed under non-SSH conditions (Figure 4a). We next tested the requirement of mTOR for synergistic gene activation, as the active mTOR fraction remaining may function under SSH. Real-time RT-PCR revealed that ICAM1 activation under SSH conditions in OVSAYO cells was diminished by treatment with the mTOR inhibitor rapamycin (Rap), which predominantly inhibits mTORC1 [5] (Figure 4b). The expression of HIFs was not influenced by

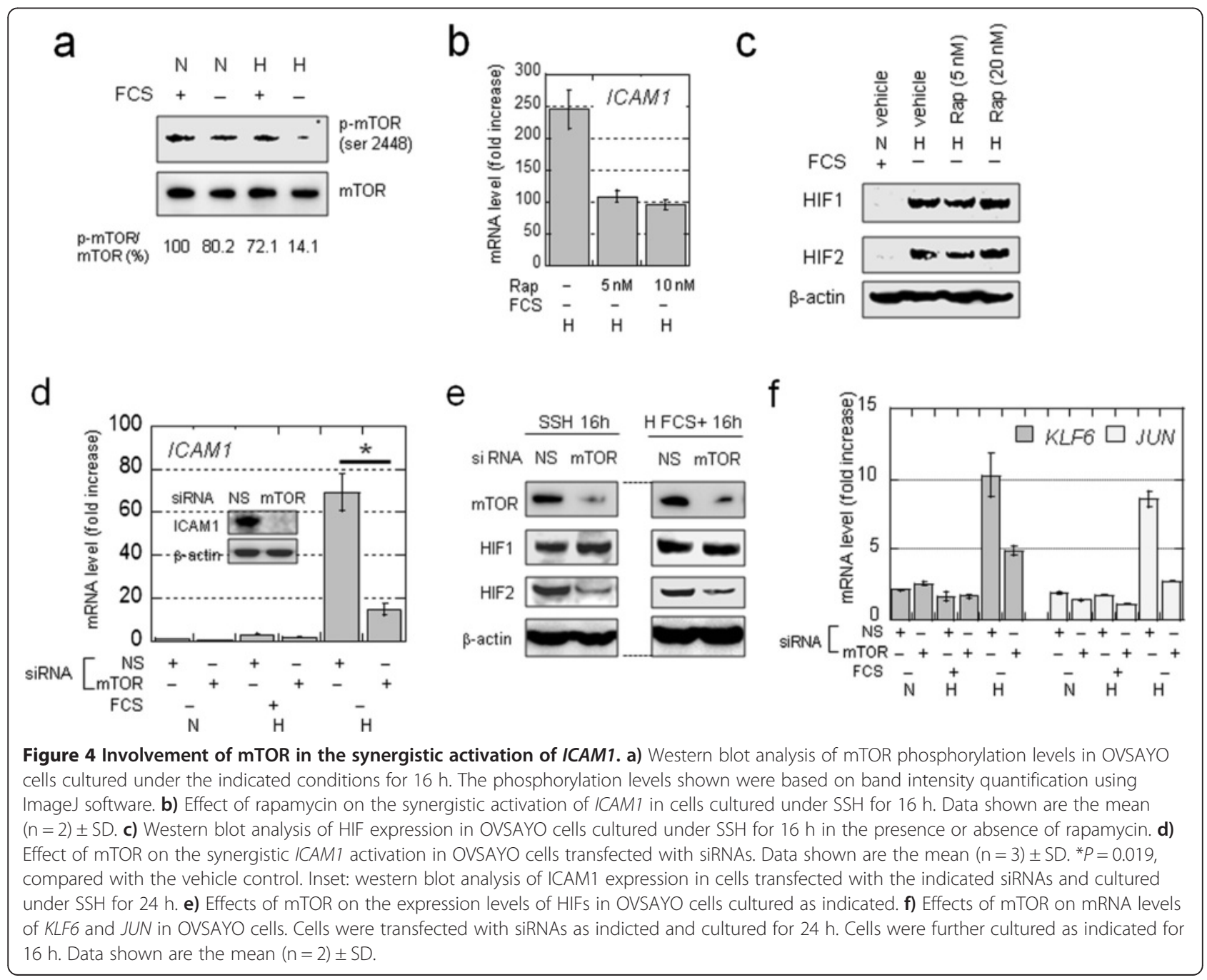


Rap treatment (Figure 4c), suggesting that the effect of Rap is not due to translational inhibition of HIFs. We also confirmed that synergistic ICAM1 activation was decreased by mTOR silencing at mRNA and protein levels (Figure 4d and e). Notably, unlike Rap treatment, HIF2 under SSH (but not HIF1) was downregulated when mTOR was silenced (Figure 4e), implying that mTORC2 is responsible for HIF2 translation [6]. However, this decrease was not responsible for inhibition of synergistic ICAM1 activation, as reduction of HIF2 levels was also seen in cells cultured with FCS (Figure 4e).

We tested the effect of mTOR on the synergistic activation of other genes. Real-time RT-PCR revealed that KLF6 and JUN induction was also repressed by mTOR silencing under SSH (Figure 4f). Furthermore, Sp1 levels in OVSAYO cells were not significantly affected by changes in the culture conditions (Additional file 2: Figure S4a). Thus, the effect of mTOR is independent of HIFs and Sp1 levels.

Activation of NFKB is crucial for synergistic ICAM1 induction $\mathrm{NF} \kappa \mathrm{B}$ is activated during inflammation, hypoxia, and irradiation and induces the expression of a variety of genes, including ICAM1 [19,25-28]. Moreover, NFkB enhances HIF1 expression at the transcriptional level [29].
However, it is unclear whether NFkB contributes to the unusual synergism of ICAM1 activation under SSH. We found that the RelA subunit of $\mathrm{NF}_{k} \mathrm{~B}$ became highly phosphorylated under $\mathrm{SSH}$ in association with $\mathrm{SSH}$ specific ICAM1 induction (Figure 5a). Western blotting of cytoplasmic and nuclear fractions revealed that RelA was translocated from the cytoplasm to the nucleus under $\mathrm{SSH}$, subsequent to the expression of HIFs (Additional file 2: Figure S4a). ChIP assays demonstrated that NFkB binding to the ICAM1 promoter (Figure $5 \mathrm{~b}$ ) is highest under SSH (Figure 5c), and real-time RT-PCR revealed that ICAM1 expression at mRNA and protein levels under SSH was markedly decreased by RelA knockdown (Figure $5 \mathrm{~d}$ ). The relative expression pattern of HIFs can be changed by RelA silencing (Figure 5e). Thus, NFKB may influence ICAM1 expression through altered HIF expression pattern. In contrast, experiments with the same RNA samples showed that KLF6 and JUN expression under SSH was not influenced by RelA silencing (Additional file 2: Figure S4b).

\section{NFKB activation under SSH involves autonomous TNFa production}

We next investigated the mechanisms involved in NFKB activation under $\mathrm{SSH}$. We first evaluated the effect of
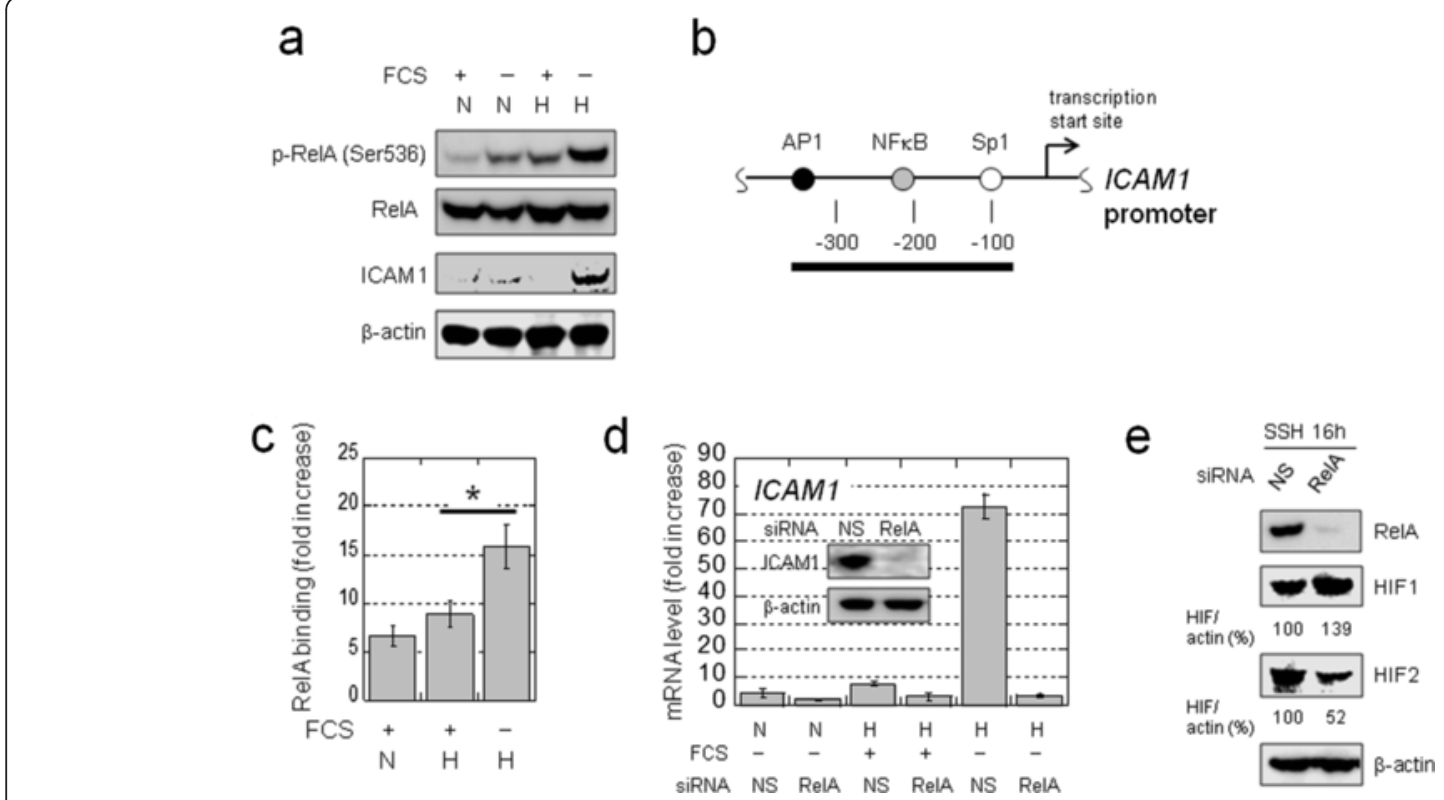

Figure 5 NFKB activation is essential for synergistic ICAM1 activation in OVSAYO cells. a) Western blot analysis of phospho-RelA (Ser536) or total RelA expression following ICAM1 induction in cells cultured under the indicated conditions for $16 \mathrm{~h} . \mathrm{N}$ and $\mathrm{H}$ indicate normoxia (20\% $\mathrm{O}_{2}$ ) and hypoxia $\left(1 \% \mathrm{O}_{2}\right)$, respectively. b) Diagram of the ICAM1 promoter region and the positions of the Sp1, NFKB, and AP1 binding sites. A black bar indicates the PCR amplicon for the ChIP assay. c) Quantitative ChIP analysis of RelA binding to the ICAM1 promoter region by real-time PCR, using cells cultured for $16 \mathrm{~h}$ under the indicated conditions. RelA binding levels are represented as fold-increases relative to negative control experiments using lgG. Data shown are the mean $(n=3) \pm S D$. ${ }^{*} P=0.02$. d) Effect of RelA on synergistic ICAM1 activation in cells cultured for $16 \mathrm{~h}$ under the indicated conditions. Data are the mean $(n=3) \pm S D$. Inset: western blot analysis of ICAM1 expression in cells transfected with indicated siRNAs and cultured under SSH for $24 \mathrm{~h}$. e) Western blotting against RelA and HIFs in lysates from cells transfected with the indicated siRNAs. HIF levels shown are based on band intensities quantified using ImageJ software. 
mTOR, as synergistic ICAM1 activation is partly dependent upon this kinase activity. Western blotting showed that RelA phosphorylation under SSH was decreased by mTOR knockdown (Figure 6a). In contrast, phosphorylation levels under hypoxia with concurrent FCS treatment were not influenced by mTOR knockdown (Figure 6a), suggesting that mTOR associates with $\mathrm{NF \kappa B}$ activity under SSH.

To explore the mechanisms of NFKB activation, we performed cDNA microarray analysis of genes synergistically activated under SSH. We found the ratio of genes with increased expression under SSH to that observed under normoxia with FCS was $>3$, while this ratio was $<2$ when comparing gene induction under normoxia without FCS and hypoxia with FCS (Additional file 2: Figure S4c and Additional file 3: Table S2). To identify potential mechanisms for these observations, we performed pathway analyses of these genes using the Kyoto Encyclopedia of Genes and Genomes database. We found that in addition to ICAM1, tumor necrosis factor- $\alpha$ (TNF $\alpha$ ) is synergistically upregulated under SSH (Additional file 2:
Figure S4d) and could potentially activate NFkB. We found that TNF $\alpha$ protein levels were low under normoxia and hypoxia with FCS stimulation, but were robustly increased in OVSAYO cells cultured under SSH (Figure 6b).

Exposure to exogenous TNF $\alpha$ can induce ICAM1 in cancer cells $[27,30]$. However, it is unclear how selfproduced TNFa affects $\mathrm{SSH}$-specific gene activation in CCC cells. Thus, we examined the effect of autonomously produced TNF $\alpha$ on the synergistic activation of ICAM1 in OVSAYO cells by real-time RT-PCR. Synergistic ICAM1 activation was reduced (Figure 6c) in OVSAYO cells in which TNFa expression was completely blocked by RNAi (Figure 6d). Notably, RelA phosphorylation levels under $\mathrm{SSH}$ were reduced by TNFa knockdown (Figure 6d), although phosphorylation under hypoxia plus FCS stimulation was not (Additional file 2: Figure S4e). The amount of HIF2 (but not HIF1) induced by SSH or hypoxia plus FCS was decreased by TNF $\alpha$ silencing (Figure $6 \mathrm{~d}$ and Additional file 2: Figure S4e). Taken together, these data indicate that mTOR expression may correlate with TNFa under SSH.
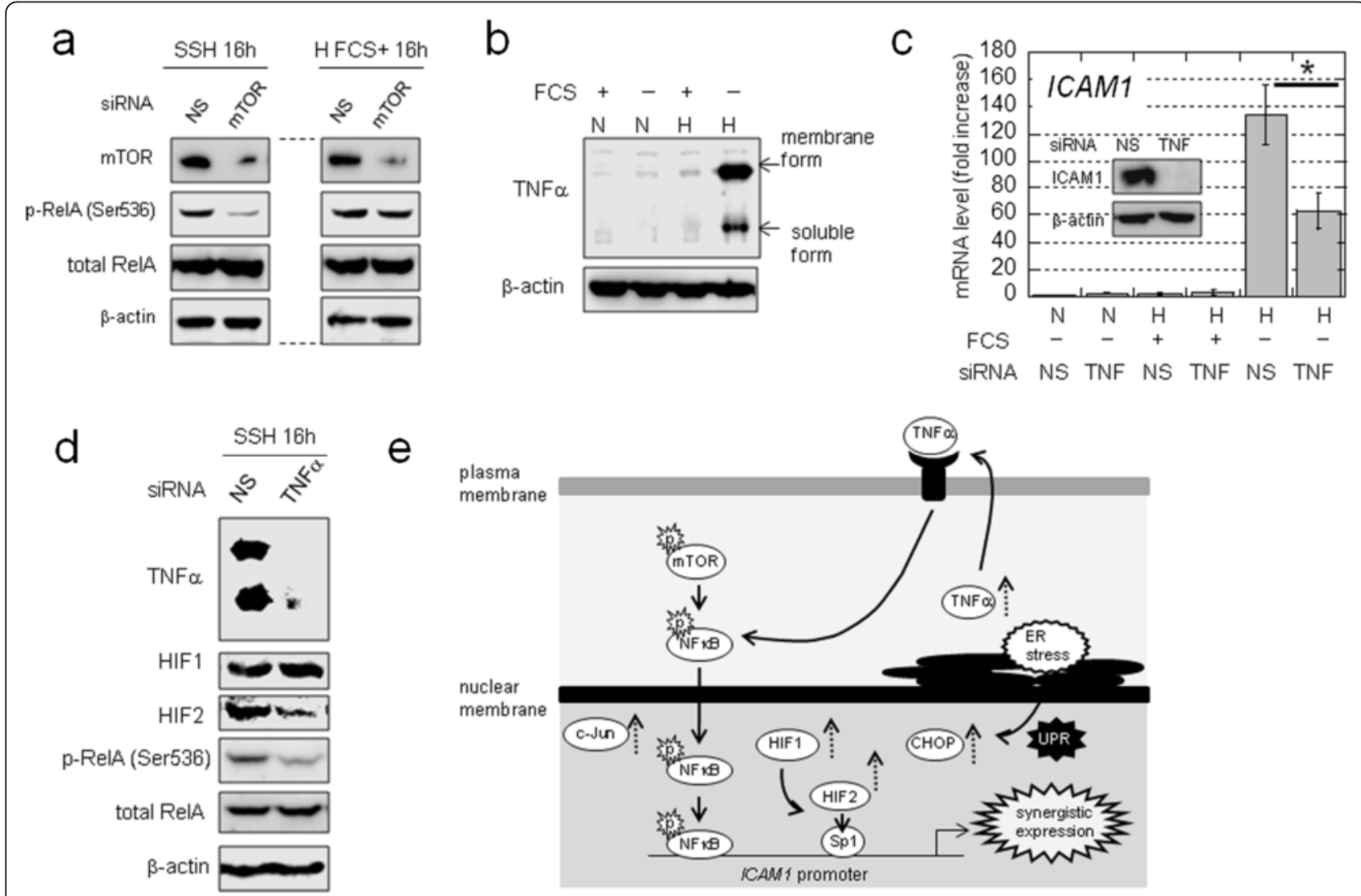

Figure 6 NFKB activation is associated with mTOR and autonomously-produced TNFa. a) Effect of mTOR on RelA activation in OVSAYO cells cultured under hypoxia. b) TNFa production in OVSAYO cells cultured under SSH for $24 \mathrm{~h}$. c) Effect of TNFa on synergistic ICAM1 activation in cells transfected with siRNAs, as indicated. Data shown are the mean $(n=3) \pm S D$. ${ }^{*} P=0.018$. Inset: western blot analysis of ICAM1 expression in cells transfected with the indicated siRNAs and cultured under SSH for 24 h. d) Effect of TNFa on RelA activation under SSH in OVSAYO cells. e) Model of ICAM1 induction under SSH. Solid arrows, dashed arrows, and " $p$ "s with bursts are indicative of the activation process, protein induction, and active (phosphorylated) states, respectively. 
We additionally tested whether synergistically induced c-Jun expression contributes to the robust expression of ICAM1, as c-Jun can regulate the ICAM1 promoter (Additional file 2: Figure S5a). Various experiments demonstrated that c-Jun does not contribute to synergistic ICAM1 activation (Additional file 2: Figure S5). Thus, we can summarize ICAM1 induction under $\mathrm{SSH}$, based on mechanisms described above (Figure 6e).

\section{Serum factors responsible for synergistic gene activation}

An unanswered question is the identity of serum factors that are responsible for synergistic gene activation. We initially found that the addition of growth factors does not suppress synergistic ICAM1 activation (data not shown). Thus, we tested whether synergistic ICAM1 induction could be repressed by the addition of albumin to serum-free culture media, as albumin is the most abundant human plasma protein and plays multiple roles in regulating blood functions [31]. We used an albumin concentration of $44 \mu \mathrm{M}$, which corresponds to protein level found in culture medium $(2.9 \mathrm{mg} / \mathrm{ml})$ with $10 \%$ FCS. We observed by real-time RT-PCR that ICAM1 mRNA levels under $\mathrm{SSH}$ were dramatically reduced in cells cultured with bovine serum albumin compared with those found without albumin (data not shown). We repeated these experiments with human albumin of differing purities. Quantitative RT-PCR results revealed that synergistic ICAM1 activation was markedly decreased in cells cultured with low purity (LP) albumin (Figure 7a). However, ICAM1 induction in cells cultured with highly purified (HP) albumin did not decrease compared with the vehicle control (Figure 7a). This albumin-dependent ICAM1 induction was also observed by RT-PCR using reduced-albumin (red-alb) serum (Additional file 2: Figure S6a and Figure 7b). However, parallel expression differences were not observed for VEGF (Figure 7b). These results suggested that LP-albumin contains serum factors responsible for suppression of the synergistic ICAM1 activation. We further showed that synergistic activation of the KLF6 and JUN genes exhibits a similar expression
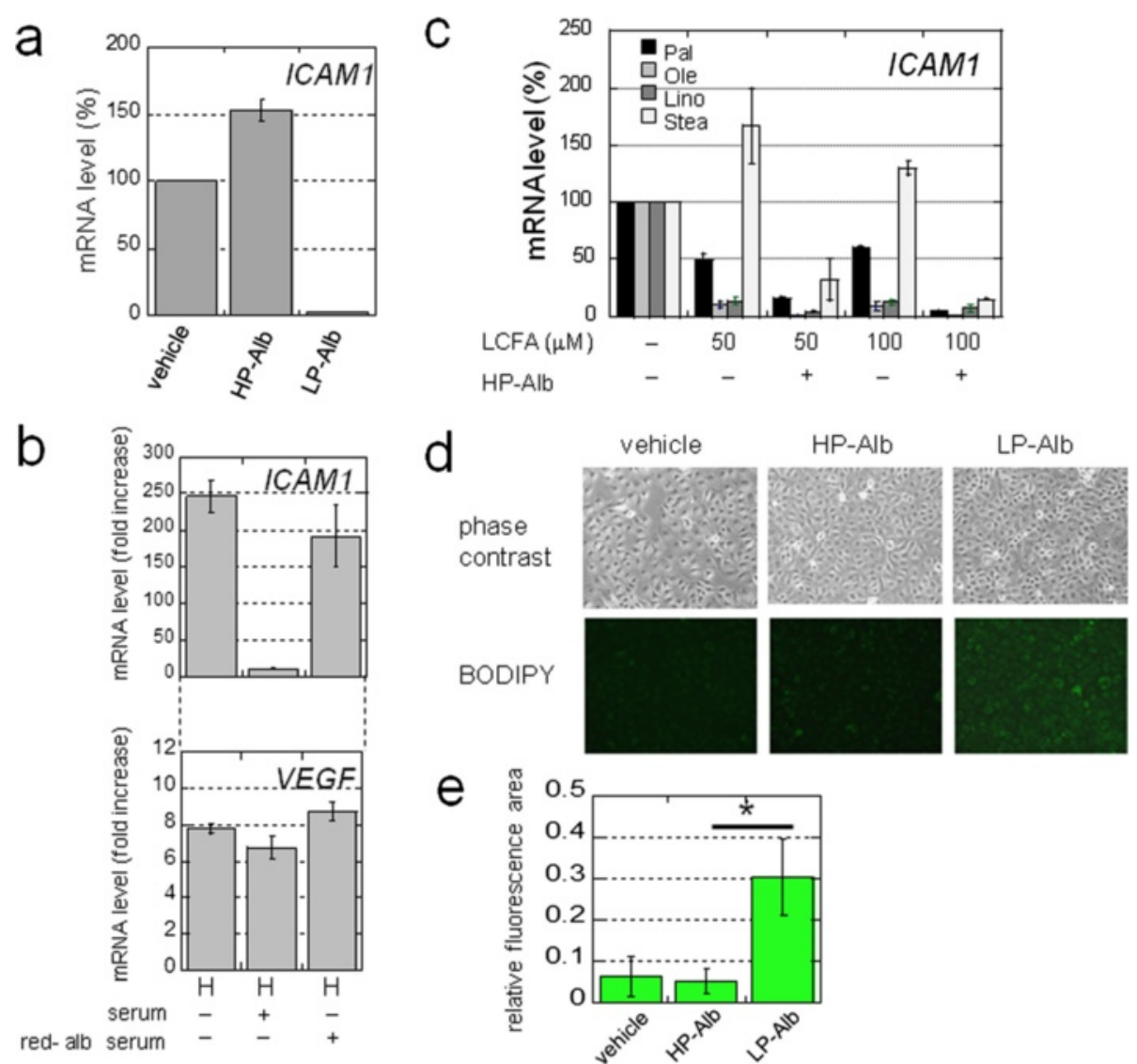

Figure 7 LCFAs are responsible for the synergistic activation of ICAM1. a) mRNA levels of ICAM1 in OVSAYO cells cultured under SSH for $16 \mathrm{~h}$ with HP- or LP-Alb. Data shown are the mean $(n=2) \pm S D$. b) ICAM1 and VEGF mRNA expression levels in cells cultured with $1 \%$ serum for $16 \mathrm{~h}$. N, normoxia $\left(20 \% \mathrm{O}_{2}\right)$; $\mathrm{H}$, hypoxia $\left(1 \% \mathrm{O}_{2}\right)$. Data shown are the mean $(\mathrm{n}=3) \pm \mathrm{SD}$. c) ICAM1 expression in cells cultured as in a) in the presence or absence of HP-Alb and various LCFAs. Data shown are the mean $(n=2) \pm S D$. d) Staining of neutral lipids in OVSAYO cells cultured as in a) with or without albumin. e) Quantitative representation of $\mathbf{d}$ ). Fluorescence areas were quantified using ImageJ software. Data shown are the mean $(n=6) \pm S D$. ${ }^{*} P=0.0002$. 
pattern in response to red-alb serum (Additional file 2: Figure S6b).

\section{Long chain fatty acids are responsible for the suppression} of synergistic gene activation under SSH

Plasma albumin binds multiple molecules including nutrients, hormones, and metabolites [31,32]. We next examined the effect of long chain fatty acids (LCFAs) on synergistic ICAM1 activation as the HP-albumin used in this study is devoid of fatty-acids. Real-time RT-PCR analysis revealed that a physiological concentration of unsaturated LCFAs [33] (oleic and linoleic acids) largely suppressed ICAM1 induction under SSH in OVSAYO cells (Figure 7c). This decrease was further enhanced in the presence of HP-albumin (Figure 7c). Weak or no suppression of ICAM1 expression was observed after exposing cells to physiological levels of saturated LCFAs (palmitic and stearic acids) alone (Figure 7c). However, ICAM1 induction was considerably suppressed when cells were simultaneously cultured with these LCFAs and HP-albumin.

We next determined the effect of LP- and HP-albumin treatment on lipid levels in cells cultured under SSH as LCFA uptake results in cytoplasmic lipid droplet accumulation in cancer cells [34]. To examine the cellular neutral lipid levels, cells were cultured in serum-free medium in the presence or absence of LP- and HPalbumin, and then stained with a neutral lipid marker, the BODIPY fluorescent dye [34]. We detected stronger BODIPY fluorescence when cells were cultured with LPalbumin (Figure $7 \mathrm{~d}$ and e), suggesting that cells are starved of neutral lipids when cultured without LCFA. This conclusion is supported by the detection of upregulated of enhanced fatty acid synthase (Additional file 2: Figure S6c), as expression of this enzyme is increased in response to cellular lipid deficiency [35].

\section{Effect of ICAM1 on CCC cell phenotype}

ICAM1 expression within poorly vascularized necrotic regions was observed in clinical CCC samples (Additional file 2: Figure S7). Thus, we next examined whether ICAM1 confers a survival advantage to ovarian cancer cells under SSH. Cloned OVISE cells stably transfected with scrambled control (Scr-) or ICAM1-shRNA were prepared. The loss of ICAM1 induction in OVISE cells under SSH was confirmed by western blotting (Figure 8a). We found that the viability of ICAM1-silenced cells under SSH was more significantly reduced compared to control shRNA cells (Figure $8 \mathrm{~b}$ and $\mathrm{c}$ ). Similar results were obtained in experiments using cell clones derived from OVSAYO cells (Additional file 2: Figure S8). Thus, ICAM1 confers a survival advantage to CCC cells exposed to $\mathrm{SSH}$.

We performed western blot analysis of PARP-1 expression to ascertain whether ICAM1 can inhibit apoptotic cell death. We detected the cleaved form of PARP-1 only in ICAM1-shRNA-transfected OVISE cells cultured under $\mathrm{SSH}$ (Figure 8d). We next examined caspase activities in shRNA-transfected cells cultured under the same SSH conditions. We found that the activities of caspases-3 and -7 were elevated in ICAM1-shRNA-transfected cells compared with control shRNA cells (Figure 8d). These results suggested that ICAM1 confers an anti-apoptotic effect under SSH.

\section{ICAM1 is induced in vivo and confers tumor growth advantage}

Some studies have shown that ICAM1 expression impairs progression of non-CCC ovarian cancers [30,36]. Thus, we further explored whether ICAM1 is induced in vivo and if so, how ICAM1 expression affects tumor growth. We first examined the proliferation of OVISE cells stably transfected with Scr-shRNAs (Scr\#1 and \#2) or ICAM1 shRNAs (ICAM1\#1 and \#2) under normoxia plus FCS stimulation and confirmed that ICAM1 knockdown caused no significant effects (Additional file 2: Figure S9a). We next subcutaneously injected Scr- and ICAM1-shRNA transfected cells into NOD-SCID mice (Figure 9a) and monitored subsequent tumor growths. We found that the growth of xenograft tumors derived from ICAM1-shRNA-transfected cells was inhibited compared with that of control cells (Figure 9b). Western blotting showed that ICAM1 levels in control tumors were higher than those of tumors with ICAM1-shRNA (Additional file 2: Figure S9b).

Hematoxylin and eosin (H\&E) staining revealed tumor tissues with typical CCC histological patterns of clear cytoplasmic and dark nuclear staining (Figure 9c). Immunohistochemistry (IHC) revealed that ICAM1 staining was stronger in control tumors than in ICAM1-silenced tumors (Figure 9d). IHC using antibodies against pimonidazole-adducts (a severe hypoxia marker, hereafter referred to as pimo-adducts) and a milder hypoxia marker (HIF1 and HIF2) revealed that the cell surface-staining pattern of ICAM1 expression (Figure 9d, e, and Additional file 2: Figure S10a) was clearly stronger within severely hypoxic areas (Figure 9e). In situ hybridization experiments provided further evidence of ICAM1 mRNA induction within severely hypoxic regions of the control tumors (Additional file 2: Figure S10b). However, ICAM1 transcripts were not detected within ICAM1-silenced tumors, although these tumor tissues were also hypoxic (Additional file 2: Figure S10c).

IHC staining further revealed that the number of Ki67-positive cells within control tumors was greater than that in ICAM1-silenced tumors (Figure 9f). However, Ki67-positive cells were poorly detected within ICAM1positive areas within tumor tissues (Additional file 2: Figure S10d and e), suggesting that ICAM1 indirectly 


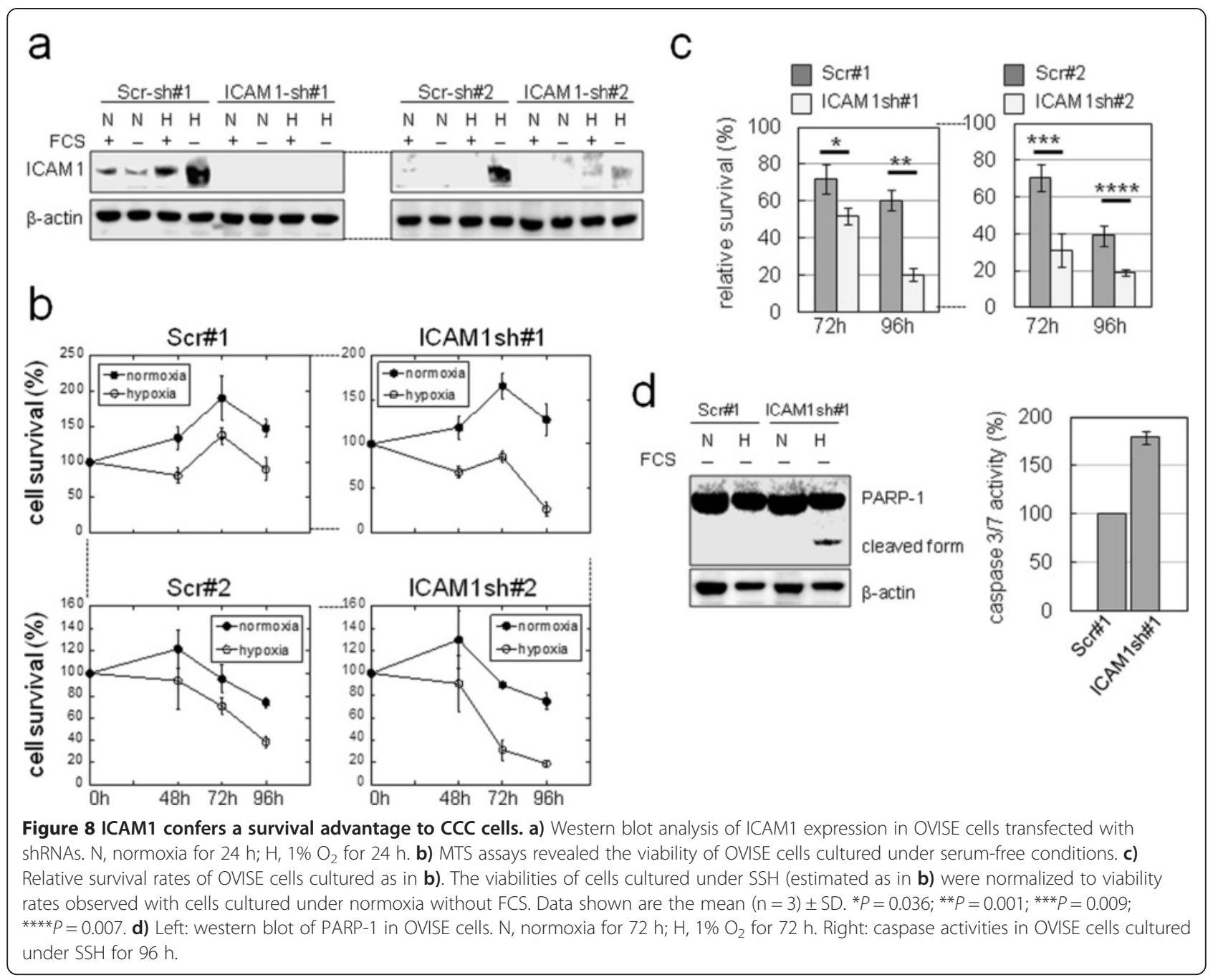

contributes to tumor growth possibly by enhancing the viability of cells.

Lipid and vessel staining revealed that tissue lipid levels were lower in vessel-poor regions in ICAM1positive xenograft tumor tissues (Figure $9 \mathrm{~g}$ and Additional file 2: Figure S11a), suggesting a directly proportional correlation between lipid contents and vessel densities. Immunofluorescence staining further showed that ICAM1 was strongly expressed within tissues in severely hypoxic regions (Additional file 2: Figure S11b).

\section{Discussion}

Here, we demonstrated that Sp1 mediates the activation of multiple genes in cooperation with HIFs. This transcriptional activation is synergistically enhanced under $\mathrm{SSH}$, in contrast to the activation of authentic HIFdependent genes. We further showed that an insufficient supply of LCFAs can lead to synergistic gene activation. The ICAM1 protein is dramatically induced by this mechanism and is critical for tumor growth in vivo, thus indicating a novel mechanism for adaptation to LCFA starvation and hypoxia as a means to promote tumor growth.

Evidence presented in this study suggests that direct binding of both HIFs and NFKB to the ICAM1 promoter region is essential for synergistic ICAM1 activation. Notably, HIF2 binding appears to be mediated via interaction with Sp1. The synergistic activation of KLF6 and JUN occurs independently of NFKB. This suggests that transcriptional cooperation between Sp1, HIFs, and NFkB promotes rigorous ICAM1 upregulation under lipid starvation and hypoxia through a distinct mechanism. In addition, the question arises as to how NFkB may be activated under $\mathrm{SSH}$, a condition where exogenous stimuli such as cytokines are absent. Our study provides 2 answers to this question. First, the activation is associated with mTOR activity. This may correlate with earlier observations that, under non-hypoxic conditions, mTOR can cause NFkB activation, leading to ICAM1 induction in cancerous and normal cells $[25,28]$. Second, NFKB can be 


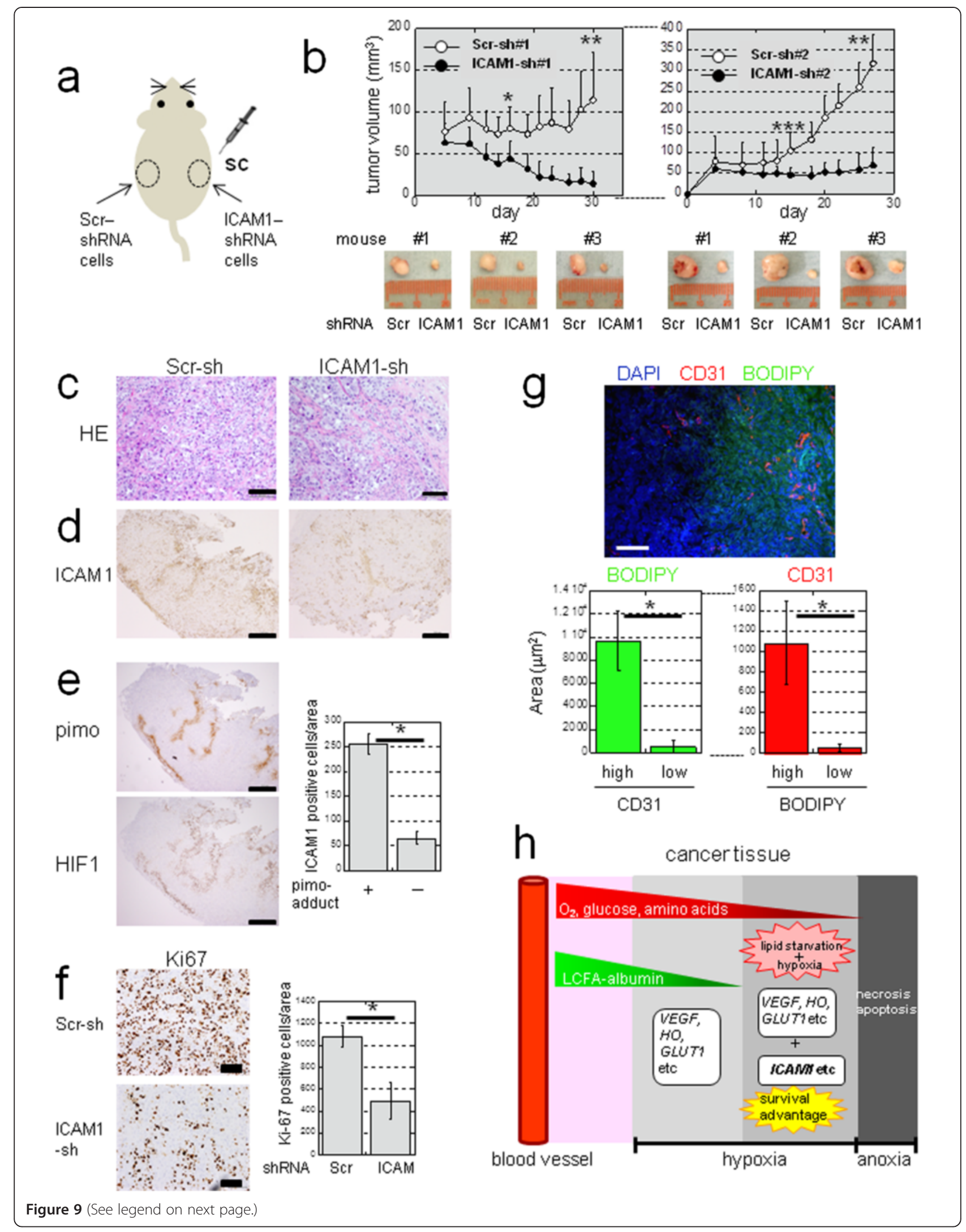


(See figure on previous page.)

Figure 9 ICAM1 is inducible in vivo and confers a growth advantage to CCC tumors. a) Sites where NOD-SCID mice were injected with cells expressing Scr-shRNA (\#1 or \#2) or ICAM1-shRNA (\#1 or \#2). b) Tumor volumes in mice injected with Scr-shRNA \#1 or ICAM1-shRNA \#1 ( $n=9$ ) or Scr-shRNA \#2 or ICAM1 shRNA \#2 $(n=10) .{ }^{*} P=0.0006 ;{ }^{* *} P<0.0001 ;{ }^{* * *} P=0.002$. c) H\&E staining (scale bar: $\left.100 \mu m\right)$. Immunohistochemistry of ICAM1 d) Pimo-adduct and HIF1 e). Quantitation of ICAM1-positive cells within pimo (+) or $(-)$ areas $(n=8)$. ${ }^{*} P<0.0001$. Scale bar: $500 \mu m$. $\left.\mathbf{f}\right)$ Immunohistochemistry of Ki67. Scale bar: $100 \mu \mathrm{m}$. Quantitation of Ki67 positive cells is shown $(n=8)$. ${ }^{*} P<0.0001$. g) Lipid staining using frozen sections of tumor tissue. Immunofluorescence of CD31 was also performed for vessels. Scale bar: $100 \mu \mathrm{m}$. The relationship between neutral lipid staining with BODIPY and CD31 positivity was quantified $(n=10)$. ${ }^{*} P<0.0001$. $\left.\mathbf{h}\right)$ Hypothetical model of lipid starvation and hypoxia associated with induction of genes of different categories in tumor tissues. Diffusion of LCFAs associated with high molecular weight albumin from the blood stream is limited compared to that of smaller molecules $\left(\mathrm{O}_{2}\right.$, glucose, and amino acids). Unlike (VEGF), (HO) and (GLUT1) genes, Sp1-dependent genes such as ICAM1 may be efficiently up-regulated only under hypoxia with a limited LCFA supply, resulting in a survival advantage of cancer cells to such a severe microenvironment.

activated by autonomously produced TNF $\alpha$ in an autocrine manner.

Recent evidence has shown that cancer cells utilize aberrant mechanisms for energy metabolism [37]. Some cancer cell types predominantly consume fatty acids rather than glucose, relying on $\beta$-oxidation for energy production $[34,35]$. In addition to their normal role as an energy source, recent studies have revealed that LCFAs may be involved in the tolerance of cancer cells to various cellular stresses [35,38]. LCFAs exist in the bloodstream in complex with albumin. It is possible that the cellular uptake of LCFAs may be facilitated only in association with albumin, resulting in their conversion to neutral lipids (acyl-CoA derivatives) [39] in CCC cells. In the present study, we showed that the removal of LCFAs synergistically enhances Sp1-dependent activation of multiple genes under hypoxia. This effect appears to be greater for unsaturated LCFAs than for saturated LCFAs. This difference may be attributed to their roles in transcriptional regulation in addition to altered metabolism pathways among LCFAs, as saturated and unsaturated LCFAs could be differentially utilized as ligands for some nuclear receptors [40]. It is necessary to uncover the detailed mechanism(s) by which CCC cells sense LCFAstarvation and hypoxia to trigger cellular signaling and induce unusually high gene expression.

We found that ICAM1 enhances cell survival under $\mathrm{SSH}$ at least in part through the inhibition of apoptosis. This is distinct from the survival mechanism whereby unsaturated LCFAs protect against UPR-dependent cell death following ischemic conditions mediated by mTOR activity [38]. Furthermore, ICAM1 promotes CCC tumor growth in mice. Previous reports have found that ICAM1 induction during inflammation enhances eosinophil survival by activating cellular signaling mechanisms, resulting in eosinophilia in asthma [41]. Thus, CCC cells might similarly activate signaling cascades via ICAM1, enabling cells to survive under severely hypoxic conditions. In additional, ICAM1 is known to be overexpressed in cancer cells where it recruits immune cells to promote tumor progression [42]. Thus, immune cell-mediated mechanisms may contribute to the acceleration of tumor growth, although it is not clear to what extent immune cells contributed in our immunodeficient mouse model. Furthermore, our data contradict earlier reports showing that ICAM1 suppresses ovarian cancer progression [30,36]. These discrepancies may be due to different histological types or experimental settings tested.

The severity of hypoxia within tumor tissues varies depending on the sparsity and aberrant nature of local vasculatures, thus creating microenvironments with limited gradients of molecules supplied from the blood. Diffusion-limited hypoxia occurs at $\sim 100-\mu \mathrm{m}$ distances from blood vessels, and hypoxic severity in tissues increases with increasing distances [43]. Given that the sizes of $\mathrm{O}_{2}$, glucose, and amino acids are smaller than that of LCFA (in complex with albumin), it is likely that areas with lipid starvation, hypoxia, expression of HIFs, and a nutrient supply exists in tumor tissues (Figure 9h). In this case, tissue lipid levels may be used to define a more severe hypoxia via Sp1 (Figure 9h). It was recently reported that immunotherapy targeting ICAM1 is effective in treating multiple myeloma [44]. Thus, further clinical investigations will likely support the validity of lipid starvation and hypoxia as a target of cancer therapy.

\section{Methods \\ Reagents}

The following reagents were obtained from Sigma (St. Louis, MO, USA): tunicamycin (T7765), HP-albumin from human serum, fatty acid-free (A3782), LP-albumin from human serum (A1653), palmitic acid (P0500), oleic acid (O1008), linoleic acid (L1376), and stearic acid (S4751). Rapamycin (553210) was purchased from (Millipore, Temecula, CA, USA).

\section{Cell culture}

Human cancer cell lines used in this study were cultured as previously described [8].

\section{Transfection with expression vectors and siRNAs}

Expression vectors and siRNAs targeting Sp1 and HIFs were described previously [12]. We employed 2 individual 
siRNA sequences targeting each target protein. See Additional file 4 for siRNAs.

\section{cDNA microarray analysis}

Total RNA was isolated as previously described [8]. cDNA microarray analysis of genes activated in response to $\mathrm{CoCl}_{2}$ stimulation in an Sp1-dependent manner was performed by Takara (Shiga, Japan), using a human whole genome array (Agilent Technologies, Santa Clara, CA, USA). The effects of serum deprivation on the expression of genes under normoxic and hypoxic conditions were analyzed by cDNA microarray analysis using a NimbleGen Catalog Eukaryotic Gene Expression $385 \mathrm{~K}$ Array (\#5543886, Roche, Madison, WI, USA). Arrays were scanned using a GenePix 4100A Microarray Scanner (Axon Instruments, Union City, CA, USA), and data were analyzed by Subio, Inc. (Kagoshima, Japan).

\section{Real-time RT-PCR analysis}

mRNA levels were determined as previously described $[8,12]$. See Additional file 4 for primer and probe sequences. The mRNA expression levels of $18 \mathrm{~S}$ ribosomal RNA were measured using SYBR green-based detection [45].

\section{Protein quantification}

Protein levels were quantified using the Micro BCA Protein Assay Kit (23235; Thermo Scientific, Rockford, IL, USA).

\section{Western blotting}

Western blotting was performed using Novex NuPAGE 4-12\% Bis-Tris gels (Life Technologies, Carlsbad, CA, USA). Detection was performed using ECL-Prime or ECL-Advance (GE Healthcare, Buckinghamshire, UK). See Additional file 4 for a description of the antibodies used.

\section{Reporter gene assays}

Reporter gene assays were performed as previously described [12]. Plasmid constructs encoding the ICAM1, KLF6, and JUN 5'promoter regions used in ChIP assays were prepared as previously described [12]. See Additional file 4 for more details.

\section{ChIP assays}

ChIP analyses were performed primarily as previously described $[8,12]$. See Additional file 4 for more details.

\section{Preparation of cytoplasmic and nuclear fractions}

Cytoplasmic and nuclear fractions were prepared using an NE-PER Nuclear and Cytoplasmic Extraction Reagents Kit (Thermo Scientific).

\section{Preparation of reduced-albumin serum}

Albumin was removed from human serum (Takara Bio, Shiga, Japan) using a Proteoprep Immunoaffinity Albumin \& IgG Depletion Kit (Sigma).

\section{Preparation of cells stably transfected with small hairpin RNAs}

Cells were stably transfected with short hairpin RNAs (shRNAs) using pcDNA6.2-GW/miR, pLenti6/V5-DEST, and the ViraPower Lentiviral Expression System (Life Technologies). Several independent clones were isolated by selecting cells in $10 \mu \mathrm{g} / \mathrm{mL}$ blasticidin. See Additional file 4 for the shRNA sequences used.

\section{Cell viability and caspase assays}

Cell viabilities were estimated in MTS assays using the CellTiter96 Aqueous One Solution Cell Proliferation Assay (Promega, Madison, IL, USA). Caspase activities were determined by performing Caspase-Glo assays (Promega).

\section{Xenograft tumor experiments}

The institutional review board at the Kanagawa Cancer Center Research Institute approved this study. OVISE $\left(1.5 \times 10^{7}\right.$ cells $)$ cell clones were subcutaneously injected into 9 (for Scr-shRNA or ICAM1 shRNA \#1) and 10 (for Scr-shRNA or ICAM1 shRNA sh\#2) NOD-SCID mice (Charles River Laboratories Japan Inc., Yokohama, Japan). Tumor growths were monitored by calculating tumor volumes as the long diameter $\times(\text { short diameter })^{2} \times 1 / 2$. A Hypoxyprobe-1 Kit (Natural Pharmacia International, Burlington, MA, USA) was used to detect tissue hypoxia. Prior to tumor isolation, mice were injected intraperitoneally with pimonidazole- $\mathrm{HCl}$ solution $(60 \mathrm{mg} / \mathrm{kg})$. At $1 \mathrm{~h}$ post-injection, mice were sacrificed under general anesthesia with isoflurane, and tumors were isolated for further experiments.

\section{Immunohistochemistry and immunofluorescence}

Routinely processed formalin-fixed, paraffin-embedded specimens were sectioned ( $4 \mu \mathrm{m}$ thickness) and stained with antibodies. Immunoreactivity was visualized by the peroxidase-labeled amino acid polymer method, using Histofine Simple Stain MAX-PO (Nichirei Co., Tokyo, Japan) and the avidin-biotin-peroxidase complex method (LSAB+; DakoCytomation Co., Tokyo, Japan), according to the manufacturer's instructions. Sections were counterstained with H\&E. In the case of ICAM1 immunofluorescence, a secondary antibody (Alexa Fluor 488 anti-mouse IgG, Life Technologies) was used. Sections were counterstained with DAPI using Prolong Gold Antifade Reagent with DAPI (Life Technologies). Antibodies against the following targets were used for $\mathrm{IHC}$ at the indicated concentrations: ICAM1 (sc-8439, $4 \mu \mathrm{g} / \mathrm{mL}$; 
Santa Cruz Biotechnology), pimo-adduct (Hypoxyprobe$1 \mathrm{Kit}, 2.3 \mu \mathrm{g} / \mathrm{mL}$; Hydroxyprobe), CD31 $(6.7 \mu \mathrm{g} / \mathrm{mL}$; Dianova GmbH, Hamburg, Germany), HIF- $1 \alpha(2.5 \mu \mathrm{g} / \mathrm{mL}$; BD Biosciences, San Jose CA, USA), HIF- $2 \alpha(10 \mu \mathrm{g} / \mathrm{ml}$; NB100-122, Novus Biologicals, Littleton, CO), and Ki67 (1:25 dilution; BD Biosciences).

\section{Microscopic detection of neutral lipid in cultured cells and tumor tissues}

Neutral lipid in cultured cells and tumor tissues was detected by fluorescence microscopy using BODIPY lipid probes (Life Technologies). Cultured OVSAYO cells were washed twice with serum-free culture media and treated with $25 \mu \mathrm{M}$ of BODIPY lipid probes in serum-free media for $5 \mathrm{~min}$ in a $37^{\circ} \mathrm{C}$ incubator. Cells were then washed twice with serum-free media and used for fluorescence detection. Images were acquired on an OLYMPUS CKX41 microscope. Fluorescence areas were quantified using ImageJ software (http://rsb.info.nih.gov/ij/). For tumor tissues, frozen sections $(8-\mu \mathrm{m}$ thickness) of xenograft tumors were treated with $100 \mu \mathrm{M}$ of BODIPY lipid probes (Life Technologies) in $50 \mathrm{mM}$ Tris- $\mathrm{HCl}$ ( $\mathrm{pH}$ 7.6) for $30 \mathrm{~min}$ at ambient temperature. Tissues were then washed twice with $50 \mathrm{mM}$ Tris- $\mathrm{HCl}(\mathrm{pH}$ 7.6), and fluorescence detection was performed. Images were acquired on a BZ-9000 fluorescence microscope (Keyence, Osaka, Japan). Quantitative analysis of images was performed using equipped BZ-Analyzer software (Keyence).

\section{Antibody labeling}

Labeling of an anti-pimo antibody with Alexa Fluor dye was performed using Zenon Mouse IgG Labeling Kits (Life Technologies), according to manufacturer's recommended procedure.

\section{In situ hybridization}

In situ hybridization was performed using an RNAscope 2.0 FFPE Assay Kit (Advanced Cell Diagnostics, Hayward, CA, USA) and an ICAM1 probe (402951, Advanced Cell Diagnostics). Routinely processed formalin-fixed paraffinembedded specimens were sectioned (4- $\mu \mathrm{m}$ thickness) and used in this study.

\section{Clinical samples}

FFPE tissues from surgically removed specimens were prepared from patients of the Yokohama City University Hospital under written agreements in the study, which was approved by our institutional review board.

\section{Statistics}

Statistical significances were evaluated using 2-sided Student's $t$-tests for 2 data sets. $P$ values $<0.05$ were considered statistically significant.

\section{Accession numbers}

The GEO database accession number for the microarray dataset is GSE55565.

\section{Additional files}

Additional file 1: Table S1. Full list of genes identified by microarray analysis described in Figure 1b. Genes appearing in this figure are underlined.

Additional file 2: Figure S1. a) ChIP analysis. b) ICAMI expression in OVISE cells. Figure S2. a) Real-time RT-PCR analysis of genes in OVSAYO cells. b) Western blotting with OVSAYO cell lysates. c) Real-time RT-PCR analysis using cells cultured under SSH. Western blotting was also performed. Figure S3. $a, b, c)$ Protein levels in OVSAYO cells. d) mRNA levels were analyzed for cells cultured with or without Tun. V: vehicle. e) Effect of Tun on the ICAM1 expression. Figure S4. a) Western analysis of indicated fractions of OVSAYO cells. b) Effect of RelA on the activation of genes. c) Venn diagram representation of genes. d) Heat map representation of gene expressions. e) Western analysis of cells transfected with siRNA. Figure S5. a) AP1 and NFKB bind to the promoter region. b) Quantitative ChIP analysis of c-Jun binding to the ICAM1 promoter. c) c-Jun expression in OVSAYO cells. d) Real-time RT-PCR analysis. Cells transfected with siRNAs were cultured under SSH. c-Jun expression in siRNA transfected cells is also shown. Figure S6. a) Approximately $80 \%$ of albumin was removed from human serum, resulting in reduced-albumin serum. b) Real-time RT-PCR analysis of indicated genes. c) Expression of fatty acid synthase (Fasn) in OVSAYO cells. Figure S7. a) ICAM1 immunocytochemistry in OVISE cells transfected with Scr-and ICAM1-shRNA \#1. Cells were cultured under SSH. b) H\&E staining and IHC using a surgically removed specimens from CCC patients. Figure S8. a) Western blot analysis, b,c) MTS assay. Figure S9. a) Proliferation of OVISE cells transfected with shRNAs. b) ICAM1 expression in tumor tissues. Figure S10. a,c,d) IHC using tumors transfected with Scr-shRNA\#2. In situ hybridization is also shown. b) In situ hybridization using tumors transfected with Scr-shRNA\#2. e) Quantitation of Ki67-positive cells. Figure S11. a) Immunofluorescence and neutral lipid staining, using tumor tissues transfected with Scr-shRNA\#2. DAPI was used for counter staining of nuclei. Bar $=100 \mu \mathrm{m}$. b) Immunofluorescence using tumor tissues transfected with Scr-shRNA\#2. Bar $=100 \mu \mathrm{m}$.

Additional file 3: Table S2. Full list of genes (probes) identified by microarray analysis as shown in Additional file 2: Figure S4C.

Additional file 4: Supplementary informations. Reagents and experimental procedures $[8,12,46]$.

\section{Competing interests}

The authors declare that they have no competing interests.

\section{Authors' contributions}

SK designed research, performed majority of experiments, analyzed data, wrote the paper. SI and RY performed animal experiments. YN and MY performed immunohistochemistry of xenograft tumor tissues. MF performed immunohistochemistry of clinical samples. EM, FH, and YT analyzed data. YM prepared cells stably transfected with shRNAs, contributed to draft the manuscript, and supervised the project. All authors read and approved the final manuscript.

\section{Acknowledgments}

This work was supported by grants to SK from the Japanese Ministry of Education, Culture, Sports, Science and Technology (Grant No. 23501280 and 26430135) and from the Yokohama Foundation for Advancement of Medical Science. We thank Naoko Yamaguchi and Tomoko Takahashi for providing technical assistance.

\section{Author details}

${ }^{1}$ Molecular Pathology and Genetics Division, Kanagawa Cancer Center Research Institute, 2-3-2 Nakao, Asahi-ku, Yokohama 241-8515, Japan. ${ }^{2}$ Departments of Pathology, Yokohama City University Graduate School of Medicine, 3-9 Fukuura, Kanazawa-ku, Yokohama 236-0004, Japan. ${ }^{3}$ Obstetrics, Gynecology and Molecular Reproductive Science, Yokohama City University Graduate School of Medicine, 3-9 Fukuura, Kanazawa-ku, Yokohama 236-0004, Japan. 
Received: 5 January 2015 Accepted: 23 March 2015

\section{Published online: 08 April 2015}

\section{References}

1. Semenza GL. Hypoxia Inducible factors in physiology and medicine. Cell. 2012;148:399-408.

2. Semenza GL. Targeting HIF-1 for cancer therapy. Nat Rev Cancer. 2003;3:721-32.

3. Koumenis C, Wouters BG. "Translating" tumor hypoxia: unfolded protein response (UPR)-dependent and UPR-independent pathways. Mol Cancer Res. 2006;:4:423-36.

4. Brown JM, Wilson WR. Exploiting tumor hypoxia in cancer treatment. Nat Rev Cancer. 2004:4:437-47.

5. Wouters BG, Koritzinsky M. Hypoxia signaling through mTOR and the unfolded protein response in cancer. Nat Rev Cancer. 2008;8:851-64.

6. Corradetti MN, Guan K-L. Upstream of the mammalian target of rapamycin: do all roads pass through mTOR? Oncogene. 2006;25:6347-60.

7. Furie B, Furie BC. The molecular basis of blood coagulation. Cell. 1988:53:505-18.

8. Koizume S, Jin M-S, Miyagi E, Hirahara F, Nakamura Y, Piao J-H, et al. Activation of cancer cell migration and invasion by ectopic synthesis of coagulation factor VII. Cancer Res. 2006;66:9453-60.

9. Yokota N, Koizume S, Miyagi E, Hirahara F, Nakamura Y, Kikuchi K, et al. Self-production of tissue factor-coagulation factor VII complex by ovarian cancer cells. Br J Cancer. 2009;101:2023-9.

10. Koizume S, Miyagi Y. Ectopic Synthesis of Coagulation factor VII in Breast Cancer Cells: Mechanisms, Functional Correlates, and Potential for a New Therapeutic Target. In: Gunduz E, Gunduz M, Rijeka, editors. Breast Cancer - Current and Alternative Therapeutic Modalities. Croatia: InTech; 2011. p. 197-212.

11. Wierstra I. Sp1: emerging roles-beyond constitutive activation of TATA-less housekeeping genes. Biochem Biophys Res Commun. 2008;372:1-13.

12. Koizume S, Ito S, Miyagi E, Hirahara F, Nakamura Y, Sakuma Y, et al. HIF2a-Sp1 interaction mediates a deacetylation-dependent FVII-gene activation under hypoxic conditions in ovarian cancer cells. Nucleic Acids Res. 2012:40:5389-401.

13. Yap TA, Carden CP, Kaye SB. Beyond chemotherapy: targeted therapies in ovarian cancer. Nat Rev Cancer. 9:167-181.

14. Tan DS, Mille RE, Kaye SB. New perspectives on molecular targeted therapy in ovarian clear cell carcinoma. Br J Cancer. 2013;108:1553-9.

15. Pages G, Pouysségur J. Transcriptional regulation of the vascular endothelial growth factor gene-a concert of activating factors. Cardiovasc Res. 2005;65:564-73.

16. Takahashi S, Matsuura N, Kurokawa T, Takahashi Y, Miura T. Co-operation of the transcription factor hepatocyte nuclear factor-4 with Sp1 or Sp3 leads to transcriptional activation of the human haem oxygenase-1 gene promoter in a hepatoma cell line. Biochem J. 2002;367:641-52.

17. Collel A, Green DR, Ricci J-E. Novel roles for GAPDH in cell death and carcinogenesis. Cell Death Differ. 2009;16:1573-81.

18. Nishi H, Nakada T, Hokamura M, Osakabe Y, Itokazu O, Huang LE, et al. Hypoxia-inducible factor-1 transactivates transforming growth factor- $\beta 3$ in troblast. Endocrinology. 2004;145:4113-8.

19. Farina AR, Cappabianca L, Mackay AR, Tiberio A, Tacconelli A, Tessitore A. Transcriptional regulation of intercellular adhesion molecule 1 by phorbol ester in human neuroblastoma cell line SK-N-SH involves Jun- and Fos-containing activator protein 1 site binding complex(es). Cell Growth Differ. 1997;8:789-800.

20. Gehrau RC, D'Astolfo DS, Prieto C, Bocco JL, Koritschoner NP. Genomic organization and functional analysis of the gene encoding the Krüppel-like transcription factor KLF6. Biochim Biophys Acta. 2005;1730:137-46.

21. Angel $P$, Hattori $K$, Smeal T, Karin M. The jun proto-oncogene is positively autoregulated by its product, Jun/AP-1. Cell. 1988;55:875-85.

22. Faber PW, van Rooij HCJ, Schipper HJ, Brinkmann AO, Trapman J. Two different, overlapping pathways of transcription initiation are active on the TATA-less human receptor promoter. The role of Sp1. J Biol Chem. 1993;268:9296-301.

23. Zhu W, Chen J, Cong X, Hu S, Chen X. Hypoxia and serum deprivation-induced apoptosis in mesenchymal stem cells. Stem Cells. 2006;24:416-25.

24. Schröder C, Witzel I, Müller V, Krenkel S, Wirtz RM, Janicke F, et al. Prognostic value of intercellular adhesion molecule (ICAM)-1 expression in breast cancer. J Cancer Res Clin Oncol. 2011;137:1193-201.
25. Ahmed M, Kundu GC. Osteopontin selectively regulates p7056K/mTOR phosphorylation leading to NF-KB dependent AP-1-mediated ICAM-1 expression in breast cancer cells. Mol Cancer. 2010;9:101.

26. Winning S, Splettstoesser F, Fandrey J, Frede S. Acute hypoxia induces HIF-independent monocyte adhesion to endothelial cells through increased intercellular adhesion molecule-1 expression: the role of hypoxic inhibition of prolyl hydroxylase activity for the induction of NF-kB. J Immunol. 2010;185:1786-93.

27. Kesanakurti D, Chetty C, Rajasekhar Maddirela D, Gujrati M, Rao JS. Essential role of cooperative NF-KB and Stat3 recruitment to ICAM-1 intronic consensus elements in the regulation of radiation-induced invasion and migration in glioma. Oncogene. 2013;32:5144-55.

28. Minhajuddin M, Bijli KM, Fazal F, Sassano A, Nakayama Kl, Hay NPlatanias LC, et al. Protein kinase C- $\delta$ and phosphatidylinositol 3-kinase/Akt activate mammalian target of rapamycin to modulate NF-KB activation and intercellular adhesion molecule-1 (ICAM-1) expression in endotherial cells. J Biol Chem. 2009:284:4052-61.

29. Majmundar AJ, Wong WJ, Simon MC. Hypoxia-inducible factors and the response to hypoxic stress. Mol Cell. 2010;40:294-309.

30. de Groote ML, Kazemier HG, Huisman C, van der Gun BT, Faas MM, Rots MG. Upregulation of endogenous ICAM-1 reduces ovarian cancer cell growth in the absence of immune cells. Int J Cancer. 2014;134:280-90.

31. Curry S. Plasma Albumin as a Fatty Acid Carrier. In: van den Vusse G, editor. Lipobiology. Amsterdam, Netherlands: Elsevier B V; 2004. p. 29-46.

32. Sugio S, Kashima A, Mochizuki S, Noda M, Kobayashi K. Crystal structure of human serum albumin at $2.5 \AA$ resolution. Protein Eng. 1999;12:439-46.

33. Glaser C, Demmelmair H, Koletzko B. High-throughput analysis of total plasma fatty acid composition with direct in situ transesterification. PLoS One. 2010;5:e12045.

34. Nieman KM, Kenny HA, Penicka CV, Ladanyi A, Buell-Gutbrod R, Zillhardt MR, et al. Adipocytes promote ovarian cancer metastasis and provide energy for rapid tumor growth. Nature Med. 2011;17:1498-503.

35. Santos CR, Schulze A. Lipid metabolism in cancer. FEBS J. 2012;279:2610-23.

36. Arnold JM, Cummings M, Purdie D, Chenevix-Trench G. Reduced expression of intercellular adhesion molecule-1 in ovarian adenocarcinomas. Br J Cancer. 2001;85:1351-8.

37. Ward PS, Thompson CB. Metabolic reprogramming: a cancer hallmark even Warburg did not anticipate. Cancer Cell. 2012;21:297-308.

38. Young RM, Ackerman D, Quinn ZL, Mancuso A, Gruber M, Liu L, et al. Dysregulated $\mathrm{mTORC1}$ renders cells critically dependent on desaturated lipids for survival under tumor-like stress. Genes Dev. 2013;27:1115-31.

39. Brasaemle DL, Wolins NE. Packaging of fat: Evolving model of lipid droplet assembly and expansion. J Biol Chem. 2012;287:2273-9.

40. van Bilsen M. Transcriptional Regulation of Cellular Fatty Acid Homeostasis. In: van den Vusse G, editor. Lipobiology. Amsterdam, Netherlands: Elsevier B V; 2004. p. 319-36.

41. Pazdrak K, Young TW, Stafford S, Olszewska-Pazdrak B, Straub C, Strarosta V, et al. Cross-talk between ICAM-1 and granulocyte-macrophage colony-stimulating factor receptor signaling modulates eosinophil survival and activation. J Immunol. 2008;180:4182-90.

42. Usami Y, Ishida K, Sato S, Kishino M, Kiryu M, Ogawa Y, et al. Toyosawa S: Intercellular adhesion molecule-1 (ICAM-1) expression correlates with oral cancer progression and induces macrophage/cancer cell adhesion. Int J Cancer. 2013;133:568-78.

43. Minchinton Al, Kyle AH. Drug Penetration and Therapeutic Resistance. In: Siemann DW, editor. Tumor Microenvironment. Oxford, UK: Wiley-Blackwell; 2011. p. 329-52.

44. Veitonmäki N, Hansson M, Zhan F, Sundburg A, Löfstedt T, Ljungars A, et al. A human ICAM-1 antibody isolated by a function-first approach has potent macrophage-dependent antimyeloma activity in vivo. Cancer Cell. 2013;23:502-15.

45. Koizume S, Yokota N, Miyagi E, Hirahara F, Nakamura Y, Sakuma Y, et al. Hepatocyte nuclear factor-4-independent synthesis of coagulation factor VII in breast cancer cells and its inhibition by targeting selective histone acetyltransferases. Mol Cancer Res. 2009;7:1928-36.

46. Koizume S, Yokota N, Miyagi E, Hirahara F, Tsuchiya E, Miyagi Y. Heterogeneity in binding and gene-expression regulation by HIF-2a. Biochem Biophys Res Commun. 2008;371:251-5. 